\title{
Pathobiology
}

\section{How a Cell Deals with Abnormal Proteins}

\author{
Pathogenetic Mechanisms in Protein Aggregation Diseases
}

\author{
A. Aigelsreiter E. Janig C. Stumptner A. Fuchsbichler K. Zatloukal H. Denk \\ Institute of Pathology, Medical University of Graz, Graz, Austria
}

\section{Key Words}

Protein folding • Protein aggregation - Chaperones •

Amyloid - Steatohepatitis, (non)alcoholic · Mallory body •

p62 $\cdot$ Keratin

\begin{abstract}
Defective protein folding is responsible for many diseases. Although these diseases seem to be quite diverse at the first glance, there is evidence for common pathogenetic principles. The basis of the pathological changes is the cell's inability to prevent protein misfolding, to revert misfolded proteins to normal or to eliminate misfolded proteins by degradation. This could result in deposition of potentially cytotoxic protein aggregates (protein aggregation diseases). Chronic degenerative diseases of the central nervous system (e.g. Alzheimer's and Parkinson's disease), the amyloidoses, but also chronic liver diseases, for example alcoholic and nonalcoholic steatohepatitis, belong to this category of disorders. This review highlights general pathogenic principles of protein aggregation diseases based on immunohistochemical and biochemical studies as well as observations in a mouse model for protein aggregation in the context of alcoholic and nonalcoholic steatohepatitis. The cellular defense mechanisms involved in protein quality control as well as the pathogenesis of protein aggregation diseases will be discussed.

Copyright $\odot 2007$ S. Karger AG, Basel
\end{abstract}

\section{Introduction}

Most proteins have to convert into a three-dimensional configuration by folding to achieve their biologically relevant function. Protein folding is a complex process susceptible to errors resulting in misfolding, aggregation, functional deficits and eventually disease. Abnormal proteins may be present in intra- and extracellular positions. Protein aggregation diseases can involve different organs and are often characterized by the appearance of cytoplasmic inclusion bodies [e.g., neurofibrillary tangles in neurons in Alzheimer's disease, Lewy bodies in Parkinson's disease (PD), inclusions in amyotrophic lateral sclerosis (ALS), Rosenthal fibers in Alexander's disease and astrocytomas, Heinz bodies in red blood cells, Mallory bodies (MBs) in hepatocytes]. The similarities in their appearance suggest common pathogenetic principles.

To understand the pathogenetic mechanisms leading to protein aggregation diseases it is important to analyze how a cell deals with abnormal proteins under physiological and pathological conditions.

\section{Protein Folding and Related Quality Control}

\section{Protein Folding}

The majority of proteins have to convert into specific tertiary structures to achieve proper biological functions [1]. Correctly folded proteins are thermodynamically sta-

\section{KARGER \\ Fax +4161306 1234 E-Mail karger@karger.ch} www.karger.com

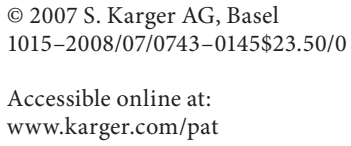

Prof. Dr. Helmut Denk, FRCPath

Institute of Pathology, Medical University of Graz

Auenbruggerplatz 25

AT-8036 Graz (Austria)

Tel. +43 316385 2228, Fax +43 316385 3432, E-Mail helmut.denk@meduni-graz.at 
ble under physiological conditions [2]. In a cell, a newly synthesized polypeptide chain can choose between three potential routes, namely nonfolding, correct folding and misfolding. The choice between low (nonfolding) and high structural complexity (folding) is determined by the amino acid sequence. Highly charged polypeptides with low overall hydrophobicity will remain in an unfolded state. However, most polypeptide chains will reproducibly acquire a specific structure at identical environmental conditions. Folding patterns, however, may be modulated by environmental pressure. Thus, also constitutively unfolded proteins may be forced to fold (or misfold) depending on their environment [3]. The folding process does not proceed through a series of mandatory steps with specific intermediates, but reflects a random search through the many conformations possible for a given polypeptide chain. The inherent fluctuations in the conformation of a polypeptide chain enable residues that are apart in the amino acid sequence to come into contact. Because interactions between residues resembling the native state are more stable than nonnative ones, they are preferred. Thus the polypeptide chain is able to find its optimal structure by a process of trial and error [2]. Due to the complexity of the folding reactions, it is obvious that errors occur quite frequently and may play a role in diseases [4]. Correct protein folding may fail because of gene mutations, RNA modifications, amino acid misincorporation during translation, or unequal synthesis of individual subunits of multisubunit protein species. Furthermore, posttranslational modifications can be caused by changes in $\mathrm{pH}$, temperature, ionic strength, and oxidative and other stresses [5]. Many misfolded proteins inappropriately expose hydrophobic domains that are normally buried in the interior of the correctly folded species. Their interaction may lead to aggregation. Aggregates can also initiate a seeding process with deposition of additional monomers and other cell components resulting in the growth of the aggregate.

Aggregates can interfere with cellular functions and are potentially cytotoxic. Three major hypotheses can explain toxicity. The first, called the channel hypothesis, suggests that ring-shaped oligomers form pores in cellular membranes; the second stresses that abnormal interactions of misfolded proteins with other proteins render the latter nonfunctional; according to the third, misfolded proteins may sequestrate components of chaperone and degradation systems, thereby reducing their activity [6].

To cope with this problem several quality control systems have evolved that act in concert to support proper folding, eliminate misfolded proteins by degradation and minimize aggregation [7].

\section{Protein Quality Control}

Molecular chaperones assist in the folding of newly synthesized proteins and are also able to promote their refolding after damage by stress and other types of cellular injury. Chaperones bind to and stabilize exposed hydrophobic domains of the target proteins and promote proper folding through cycles of controlled binding and release [5, 8]. On the other hand, cellular proteins, which are unable to fold or refold properly, are degraded by the ubiquitinproteasome system. Finally, when correct folding is impossible and degradation by the ubiquitin-proteasome system not sufficient, the abnormal proteins aggregate and accumulate as inclusion bodies (figs. 1, 2) $[9,10]$.

\section{Chaperones}

Chaperones are ubiquitous, highly conserved proteins. The major classes are listed in table 1 [11-13]. Their functions in addition to assisting proper protein folding includes stimulation of assembly and disassembly of multimeric proteins, and facilitation of protein translocation across the membranes of the endoplasmic reticulum (ER), mitochondria, peroxisomes and nuclei [14]. Moreover, chaperones are also required for degradation of abnormal and certain normal, short-lived proteins in both prokaryotes and eukaryotes by being involved in three degradation pathways, namely mitochondrial, ubiquitin-proteasomal, and lysosomal proteolysis [14]. The link between folding and degradation processes is provided by the proteins CHIP and BAG1. CHIP acts as an ubiquitin ligase which associates with chaperones and polyubiquitinates their substrates, whereas BAG1 links heat shock protein (Hsp) 70 with the $26 \mathrm{~S}$ proteasome thus facilitating delivery of Hsp70-bound targets to the proteasome [15].

When protein damage occurs, e.g., due to heat stress, $\mathrm{pH}$, certain chemicals or metals that affect their folding properties, proteins expose hydrophobic domains on their surface, which are then recognized by chaperones [16]. In order to protect the cell during stress, chaperones are upregulated. Therefore, most chaperones are also called stress protein or after the archetype of experimental stress, namely high temperature, Hsps [17]. Their action may be ATP-dependent or ATP-independent. The activities of the two major high-molecular-weight Hsp70 and Hsp90 are regulated by ATP binding and hydrolysis, whereas lowmolecular-weight small Hsps (sHsps), like Hsp27, act in an ATP-independent fashion [18]. Both types cooperate: sHsps bind unfolded proteins and serve as protector 'resting places' for proteins during stress conditions until ATPdependent high-molecular-weight $\mathrm{Hsps}$ are available to assist folding [19]. Clusterin action resembles that of sHsps; 
Fig. 1. Molecular chaperones assist in the folding of newly synthesized proteins. The off-folding pathway comprises three routes: (1) the ubiquitin-proteasome pathway that competes for and degrades offpathway intermediates before they can aggregate, (2) the amorphous aggregation pathway with final irreversible precipitation, and (3) the slower amyloid fibrilforming pathway that leads via formation of small soluble protofibrils to insoluble highly ordered cross $\beta$-fibrils.

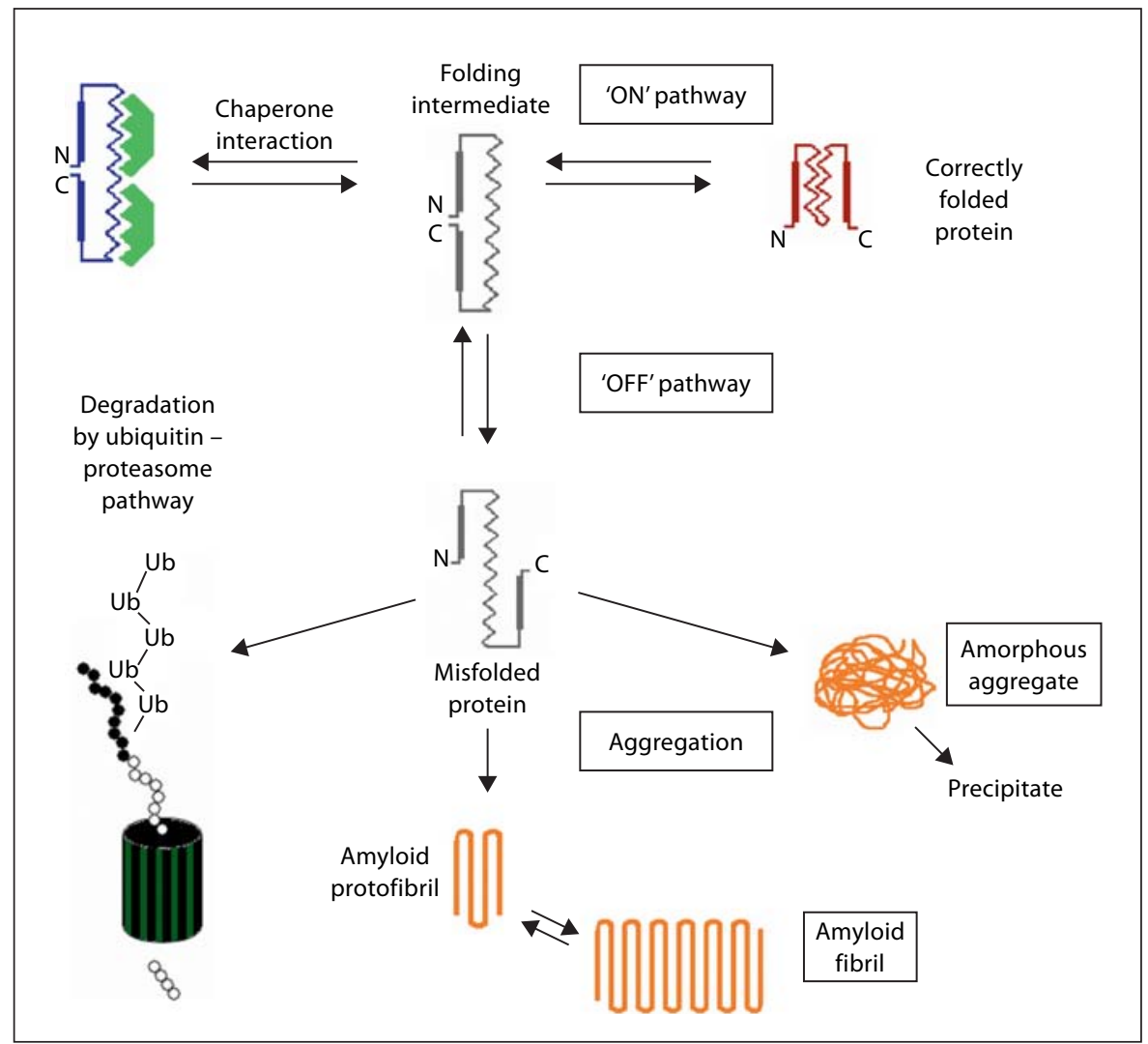

Table 1. Major molecular chaperone families

\begin{tabular}{lll}
\hline Chaperone & Function & Localization \\
\hline sHsp (Hsp27, crystallins) & Prevents protein aggregation and keeps unfolded proteins in solution & Intracellular \\
Hsp60, chaperonins & Prevents protein aggregation, assists protein folding & Intracellular \\
Hsp70, Grp 78, BiP & Prevents protein aggregation, assists protein folding & Intracellular \\
Hsp90, Grp 94 & Prevents protein aggregation & Intracellular \\
Hsp110 & Releases proteins from aggregates & Intracellular \\
Clusterin & Prevents protein aggregation & Extracellular
\end{tabular}

it also binds to exposed hydrophobic regions of unfolded proteins and inhibits aggregation by stabilizing them in an ATP-independent manner. However in contrast to other sHsps, clusterin is secreted and represents the first identified chaperone of the extracellular space [20].

The protein folding pathway is reversible and, under stress conditions, proteins may unfold or adopt intermediately folded states (fig. 1). Two scenarios are possible: (1) deviation from the normal folding/unfolding to an irreversible off-folding pathway, leading to aggregation and precipitation or (2) interaction with Hsps. In the latter situation kinetic factors play an important role in the efficiency of chaperone action. sHsps prefer to interact with slowly aggregating proteins; in contrast, $\mathrm{Hsp} 70$ recognizes intermediately folded states of target proteins that are present only transiently on the rapid protein unfolding/ folding protein pathway [13]. The off-folding pathway comprises three routes: (1) the ubiquitin-proteasome pathway that degrades off-pathway intermediates before they can aggregate, (2) the amorphous aggregation pathway with irreversible precipitation, and (3) the amyloid fibril forming pathway that leads via formation of small 
soluble protofibrils to insoluble highly ordered cross- $\beta$ fibrils, consisting of polypeptide chains in an extended beta conformation (fig. 1) $[9,13]$.

Like the 'heat shock response' in the cytoplasm during stress situations the 'unfolding protein response' in the ER is also upregulated. Cellular secretory proteins are folded in the ER prior to secretion through the Golgi apparatus. Many different molecular chaperones and folding catalysts promote efficient folding in the ER. This process involves a complex series of glycosylation and deglycosylation reactions. Misfolded proteins are not secreted, but transported into the cytoplasm and targeted for degradation by the ubiquitin-proteasome pathway [1].

\section{Ubiquitin-Proteasome System}

Mammalian cells contain several protein degradation systems including lysosomal proteases, calcium-dependent proteases, the proteasome, and the mitochondrial Lon protease [21]. The major nonlysosomal degradation route is the ubiquitin-proteasome pathway [22]. The proteasome in the eukaryotic cell eliminates polyubiquitinconjugated proteins by degrading them into small peptides. The proteasome is also present in numerous prokaryotic organisms, whereas the ubiquitin-conjugating system is absent. In eukaryotes the proteasome not only eliminates proteins, but also participates in diverse other vital cellular processes such as the cell cycle, differentiation, regulation of circadian rhythms, long-term memory, DNA repair, transcriptional silencing, antigen presentation, and in the defense against neoplastic transformation and the cellular interaction with viruses [23]. Proteasomemediated degradation is mostly ubiquitin-dependent. To be degraded, proteins have to be conjugated with a polyubiquitin chain. Ubiquitination is a highly ordered process in which enzymes activate and transfer ubiquitin to the target substrate [22]. Three types of enzymes are involved: the ubiquitin-activating enzyme E1, the ubiquitin-conjugating enzyme E2, and the ubiquitin ligase E3 [24].

Ubiquitination is essential to be recognized by diverse polyubiquitin binding proteins. Two related families of polyubiquitin binding proteins guide the ubiquitinated proteins to the proteasome or to the ATPase complex Cdc 48. These two act sequentially in the degradation process [25]. The proteasome holoenzyme, the $26 \mathrm{~S}$ proteasome, consists of two major subcomplexes: the 20S core particle, which contains the protease subunits, and the $19 \mathrm{~S}$ regulatory particle (RP), which regulates the function of the former [26]. The RP can be further dissected into two multisubunit substructures, a lid and a base, which contains an ATPase domain [23]. ATP is initially involved in the acti- vation of ubiquitin, which is then transferred to one of the 20-40 different ubiquitin-carrier proteins (E2). The selectivity of this action resides in the ubiquitin ligases (E3), which are specific for different protein substrates. There is a multitude of different ubiquitin ligases, which together with a specific E2 catalyze the attachment of the ubiquitin chain to protein substrates. Finally, rapid degradation by the $26 \mathrm{~S}$ proteasome occurs [26]. Besides the proteasome, the ATPase complex Cdc 48 is involved in the process. In contrast to the proteasome, Cdc 48 lacks an 'overhanging structure' which precludes the access of larger proteins to the unfolding site of the proteasome. It is therefore likely that the $\mathrm{Cdc} 48$ is capable of unfolding bulky ubiquitinated proteins more readily, converting them into a state that is more suitable for the proteasome [25].

\section{Protein Aggregation}

A novel cytoplasmic structure, the aggresome, has been described by Kopito [9] and Johnston et al. [27]. Aggresomes form when a cell's capacity to degrade misfolded proteins is exceeded. Aggresomes can be experimentally induced in tissue culture cells by overexpression of aggregation-prone protein or inhibition of proteasome acivity $[27,28]$. Aggregated proteins accumulate in a pericentriolar structure within a collapsed intermediate filament (IF) network [27]. The fact that aggregated proteins are concentrated close to the microtubule-organizing center and that this process requires an intact cytoskeleton led to the model of retrograde microtubular transport of misfolded proteins [28]. It has been proposed that aggresome formation provides a 'staging ground' for the incorporation of protein aggregates into autophagic structures, possibly by facilitating interaction with endosomes and lysosomes, which are also delivered by microtubules to the same region of the cell [27].

Another novel cytoplasmic compartment, at which excess ubiquitinated proteins are segregated, has been designated sequestosome by Shin [10]. He found that the nonproteasomal multiubiquitin chain binding protein sequestosome 1/p62 (p62) is a major component of the sequestosome and concluded that the sequestosome is involved in the regulation of the fate of multiubiquitinated proteins. In contrast to the aggresome the sequestosome is not located at the pericentriolar region. Sequestosome formation is enhanced when proteasomal activity is impaired, suggesting a storage function. It might be that with decreased proteasomal activity, ubiquitin-protein conjugates segregate into the sequestosome, being then released upon proper signals for proteosomal degradation [10]. 


\section{Abnormalities of Protein Folding and Associated Diseases (Protein Aggregation Disorders)}

A variety of diseases can be attributed to defective protein folding. The basis of the pathological changes is the inability of the cell to prevent protein misfolding or to degrade misfolded proteins with the consecutive formation of potentially cytotoxic protein aggregates. The etiology of protein aggregation diseases is multifactorial with genetic as well as environmental facets. Besides amino acid alterations due to mutations, elevated temperature, $\mathrm{pH}$ changes, viral infections and oxidative stress contribute to the pathogenesis of these disorders. Diseases characterized by abnormal proteins due to amino acid alterations caused by gene mutations comprise three groups. (1) Autosomal recessive disorders with loss of function, such as cystic fibrosis or phenylketonuria. In this group the mutation causes prolonged interaction of the protein with the chaperone, resulting in its functional deficiency. The phenotype depends on the degree of misfolding in response to different types of mutations. (2) Autosomal dominant disorders with gain of dominant negative function, such as keratin and collagen diseases and familial forms of cardiomyopathies. The misfolded proteins are not degraded and inhibit the normal function of the respective protein. The phenotype can again be mild or severe depending on the nature and position of the mutations. (3) Diseases with 'toxic' aggregation-type gain of function, such as amyloidoses and late-onset neurodegenerative disorders. The mutant proteins form insoluble oligomers and polymers/aggregates, which exert a toxic gain-of-function effect on the cell. The clinical phenotype results from cell damage and death.

Oxidative stress is the prototype of nongenetic causes of protein misfolding and contributes to the pathogenesis of many conformational diseases. Reactive oxygen species that damage the cell are particularly generated when the mitochondrial oxidative phosphorylation and the cell's antioxidative capacity become overwhelmed. Reactive oxygen species damage DNA, RNA, lipids and proteins. Misfolded and partly unfolded proteins are especially susceptible to oxidative modifications with consecutive unfolding and further aggregation. Defects in the mitochondrial respiratory chain have been found in brain cells of patients with neurodegenerative disorders. Furthermore, in aged cells the resistance to oxidative stress is decreased and the capacity for protein quality control reduced [19].

\section{Amyloidosis}

The term 'amyloid' was originally coined by Virchow in 1854, because of its starch-like staining with iodine.
Later its protein nature was discovered. Today amyloid is defined as a protein in a specific fibrillar state. Characteristics are (1) its green birefringence under polarized light after Congo red staining, (2) its fibrillar appearance under the electron microscope, and (3) its $\beta$-sheet X-ray diffraction pattern [29]. To date at least 21 different proteins have been recognized to be involved in amyloid formation (= amyloidogenic proteins). Although they differ in structure and function, they assemble to morphologically indistinguishable amyloid fibrils [30]. These fibrils consist of polypeptide chains in an extended beta conformation, hydrogen-bonded together as sheets which run parallel to the axis of the fibril, forming the so-called 'cross-beta conformation' [29]. Amyloid fibrils seem to be dynamic structures and studies suggest recycling of half of the molecules within an assembly of fibrils with an average length of $100 \mathrm{~nm}$ between 2 and 20 days. These new insights provide the basis for therapeutic approaches by interfering with fibril assembly [31].

Systemic, organ-limited and intracellular types of amyloidosis can be distinguished [32], as presented in table 2 .

With regard to systemic amyloidosis there is a growing list of secreted, circulating proteins that are capable of producing extracellular amyloid deposits in multiple organs [33]. Accumulation of serum amyloid A (SAA) may be associated with chronic inflammatory disorders, like rheumatoid arthritis or tuberculosis [32]. Multiple myeloma causes overproduction and deposition of amyloid consisting of immunoglobulin light chains and their fragments $[33,34]$. There are also therapy-related forms. For example, chronic hemodialysis leads to progressive increase in $\beta_{2}$ microglobulin in tissues because the protein cannot pass efficiently through the dialysis membrane [35]. The familial Mediterranean fever is associated with deposition of SAA protein. In the senile systemic amyloidosis wild-type transthyretin accumulates [32]. In organ-limited amyloidosis the protein accumulates locally in the extracellular space near the site of its production. Examples are deposition of calcitonin in medullary thyroid carcinoma, of amylin in the Langerhans islets in type II diabetes mellitus, or of atrial natriuretic factor in atrial tissue of the heart.

In contrast to extracellular amyloidosis intracellular amyloid deposits are usually noncongophilic and thus differ in their staining behavior from classical extracellular amyloid. Several chronic neurodegenerative diseases belong to this category. They include Alzheimer's disease, PD, frontotemporal dementia with parkinsonism, Creutzfeldt-Jakob disease (CJD), Huntington's disease, spinocerebellar ataxia [32] and ALS [36]. 


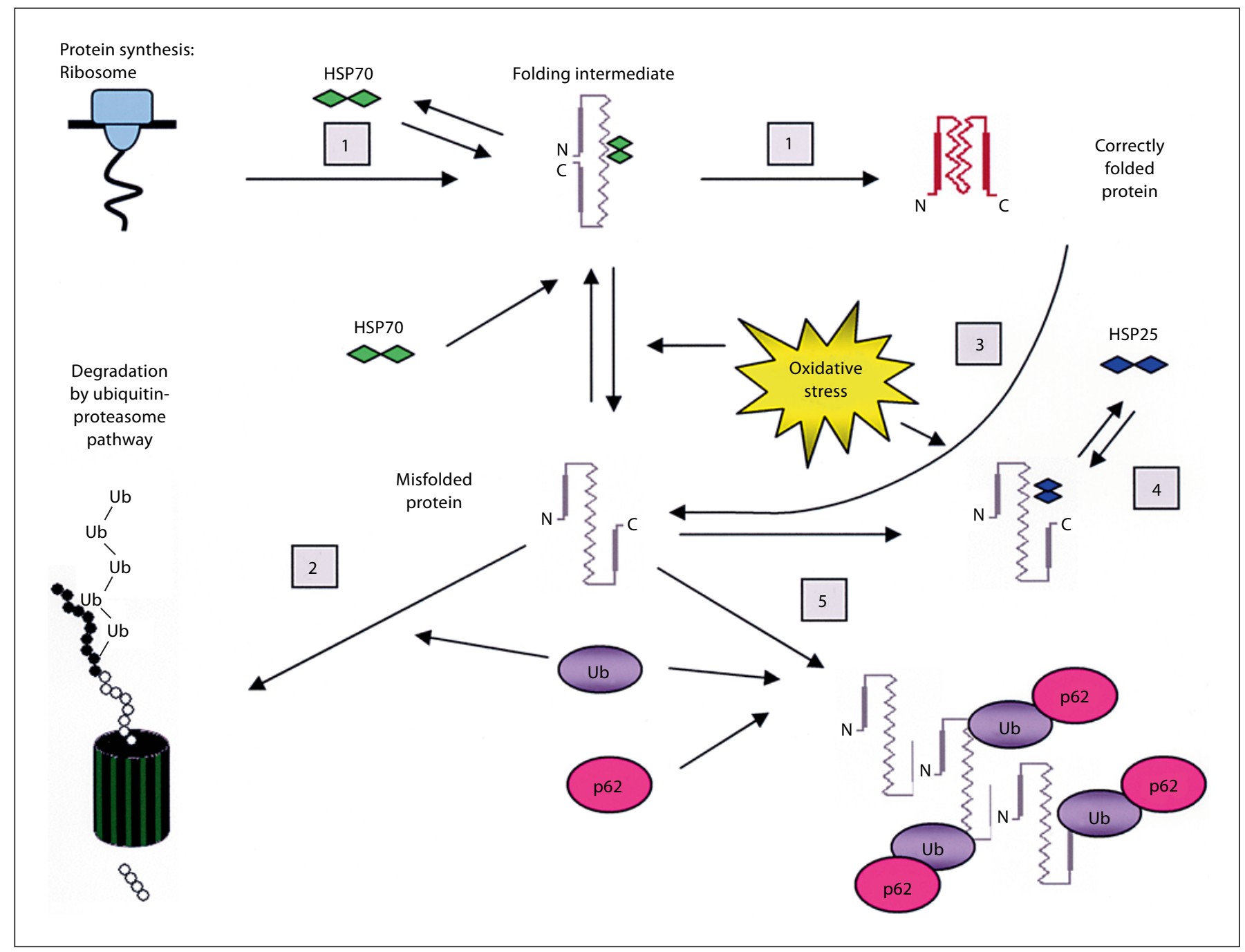

Fig. 2. (1) Heat shock proteins (Hsp70) assist in the folding of newly synthesized proteins (e.g., keratins). (2) Misfolded proteins are ubiquitinated and degraded by the ubiquitin-proteasome pathway. (3) When protein folding is impaired by oxidative stress partially folded proteins can refold with the assistance of Hsp70. (4) sHsp (sHsp25) bind unfolded proteins and serve as 'resting places' for proteins during stress conditions until ATP-dependent high-molecular-weight Hsps (Hsp70) are available to assist folding. (5) If in chronic stress situations the cell's proteolytic capacity is impaired and the degradation by the ubiquitin-proteasome pathway unable to keep up with the production of abnormal proteins (e.g., keratins), they aggregate, e.g., as MBs, together with p62 and ubiquitin.

\section{Neurodegenerative Diseases}

The main characteristics of these diseases are their chronic and progressive course with selective and symmetric loss of neurons in either the motoric, the sensory, or the cognitive system [37]. The region affected determines the clinical symptoms [38]. Another mandatory feature is the mostly intracellular deposition of amyloid material, with the exception of Alzheimer's disease in which the intracellular and the extracellular space is involved [32]. Inflammation (reactive astrocytosis and ac- tivated microglia) is also present [38]. These diseases may be inherited or sporadic. Huntington's disease is almost always inherited, whereas Alzheimer's disease, PD and ALS are only inherited in 1-10\% of the cases [37]. Sporadic forms are mostly associated with aging, where oxidative modifications of proteins play a major role [39].

\section{Alzheimer's Disease}

The most common type of neurogenerative diseases is Alzheimer's disease. Morphologic hallmarks are neurofi- 
Table 2. Protein aggregation diseases

\begin{tabular}{|c|c|c|c|}
\hline Disease & Protein & Inclusion (designation) & Localization \\
\hline \multicolumn{4}{|l|}{ Chronic inflammatory disorders } \\
\hline (e.g. rheumatoid arthritis, tuberculosis) & SAA protein & - & Extracellular \\
\hline Multiple myeloma & Immunoglobulin light chains & - & Extracellular \\
\hline Chronic hemodialysis & $\beta_{2}$-Microglobulin & - & Extracellular \\
\hline Familial Mediterranean fever & SAA protein & - & Extracellular \\
\hline Senile systemic amyloidosis & Wild-type transthyretin & - & Extracellular \\
\hline Medullary thyroid carcinoma & Calcitonin & - & Extracellular \\
\hline Type II diabetes mellitus & Amylin & - & Extracellular \\
\hline \multirow[t]{2}{*}{ Alzheimer's disease } & Amyloid fibrils & Amyloid plaques & Extracellular \\
\hline & Tau, p62 & Neurofibrillar tangles & Intracellular \\
\hline $\mathrm{PD}$ & Alpha synuclein, p62 & Lewy bodies & Intracellular \\
\hline Amyotrophic lateral sclerosis & SOD1 & Bunina bodies & Intracellular \\
\hline Huntington's disease & Huntingtin & - & Intracellular \\
\hline Prion disease & Prion protein $\left(\mathrm{PrP}^{\mathrm{SC}}\right)$ & - & Intracellular \\
\hline$\alpha_{1}$-AT deficiency & $\alpha_{1}-\mathrm{AT}$ & - & Intracellular \\
\hline $\begin{array}{l}\text { ASH, NASH, copper toxicosis, hepatocellular } \\
\text { carcinoma, Indian childhood cirrhosis }\end{array}$ & K8, K18, p62, ubiquitin & Mallory bodies & Intracellular \\
\hline
\end{tabular}

brillar tangles consisting of paired helical filaments (PHF) within neuronal cell bodies and neuritic processes. Paired helical filaments are mainly composed of the microtubule-associated protein tau. The other major morphologic hallmarks in Alzheimer's disease brains are the amyloid plaques. These are extracellular deposits of insoluble amyloid fibrils resulting from neuronal damage. The extracellular chaperone clusterin is associated with amyloid plaques in Alzheimer brains [40] (fig. 3a). In experimental studies, clusterin inhibits the aggregation of $\beta$-amyloid protein, which indicates its possible neuroprotective role [41]. Amyloid plaques surrounded by dystrophic dendrites and axons are called 'neuritic plaques', which are localized in the dentate gyrus of the hippocampus, the amygdala, the association cortices of the frontal, temporal and parietal lobes, and certain deep brain nuclei [42]. Recently the stress and adapter protein p62 could be identified as a novel component of neurofibrillar tangles (fig. 3b) as well as in Lewy bodies in PD (fig. 3c) $[43,44]$.

\section{Parkinson's Disease}

$\mathrm{PD}$ is characterized by selective degeneration of dopaminergic and noradrenergic neurons in the substantia nigra and the locus ceruleus. In idiopathic PD the neurons harbor spheroidal inclusions called Lewy bodies. Lewy bodies consist of $\alpha$-synuclein and are formed under the influence of oxidative cell stress. Idiopathic PD may be triggered by chronic exposure to environmental toxins (for example rotenone or paraquat) that can inhibit mito- chondrial complex I and promote free radical stress. In familial PD the most common mutated gene is parkin. Parkin is a cytoplasmic neuronal protein that functions as an E3 ligase. Due to this mutation an aggressive form of $\mathrm{PD}$ arises in which dopaminergic midbrain neurons are lost; surviving cells lack Lewy bodies [42].

\section{Amyotrophic Lateral Sclerosis}

This disease is characterized by progressive degeneration and loss of motor neurons with astrocytosis. It is fatal since an effective therapy is still lacking. $5-10 \%$ of ALS are familial, the rest is sporadic. Approximately $20 \%$ of familial ALS cases are associated with superoxide dismutase 1 (SOD1) mutations, the remaining are caused by mutations of other genes including neurofilament-related ones. In degenerating neurons and glial cells various cytoplasmic inclusions can be seen, such as Bunina bodies in about $70 \%$ of autopsied patients, ubiquitinated inclusions, Lewy-like bodies, conglomerate hyaline inclusions (in some patients with familial ALS), and advanced glycated end products, deposited by a process of glycation and oxidation (in familial ALS with the A4V mutation in the gene for SOD1) [36]. An immunohistochemical analysis revealed p62 as a component of intraneuronal inclusions in ALS associated with dementia [45].

\section{Huntington's Disease}

This disease, inherited in an autosomal-dominant trait, is associated with progressive chorea and dementia 


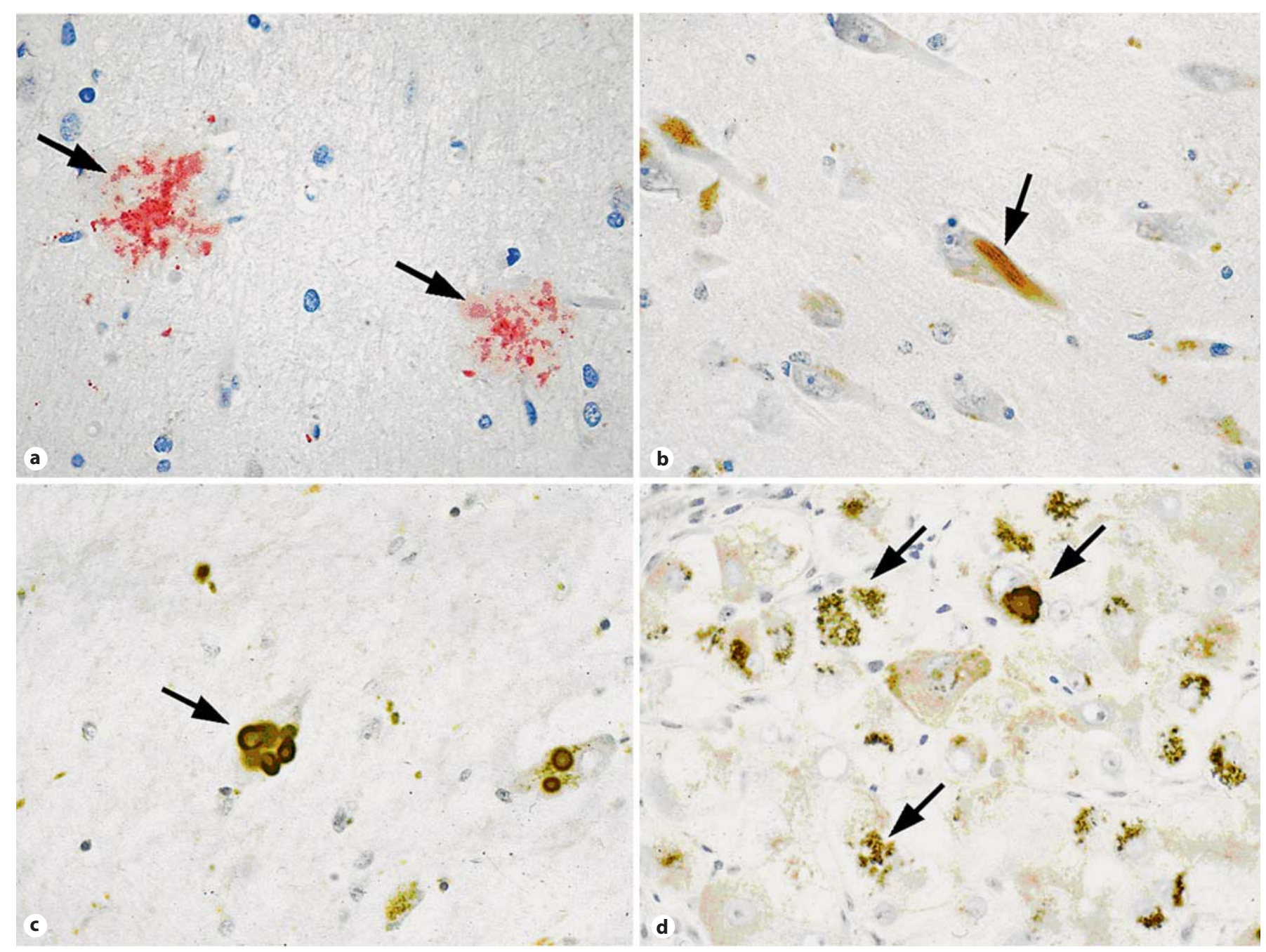

Fig. 3. a Alzheimer's disease: immunohistochemical staining for clusterin. Arrows indicate the presence of clusterin in extracellular amyloid plaques. b Alzheimer's disease: immunohistochemical staining for p62. Arrow indicates the presence of p62 in neurofibrillar tangle. c PD: immunohistochemical staining for p62. Arrow indicates the presence of p62 in Lewy body. $\mathbf{d} \alpha_{1}$-AT deficiency: immunohistochemical staining for $\alpha_{1}$-AT in hepatocytes. Arrows indicate inclusion bodies. a-d $\times 200$.

caused by severe neuronal loss in the neostriatum and later in the cerebral cortex. The Huntington's disease (HD) gene encoding for the protein huntingtin contains an unstable CAG repeat in the open reading frame of its first exon. The increased number of CAG repeats leads to elongated glutamine residues in the huntingtin protein, which favor aggregation. The resulting intraneuronal inclusions consist of huntingtin and ubiquitin and are found in the striatum and cerebral cortex [37]. Increased expression of p62 was found in association with polyglutamine inclusions in Neuro2a cells suggesting a role of this protein in response to polyglutamine-induced stress [46].

\section{Prion Disease}

Prion diseases include scrapie in sheep, bovine spongioform encephalopathy (BSE) in cattle and CJD in humans. Accumulation of the pathological prion protein $\left(\mathrm{PsP}^{\mathrm{SC}}\right)$ in the central nervous system is a common disease feature. Furthermore, these diseases are characterized by long periods of latency before overt degeneration of the central nervous system $[47,48]$. The majority of CJD cases $(85 \%)$ are sporadic without defined etiology. Acquired forms are less frequent and include iatrogenic and variant CJD, which has been linked to infection with the agent causing bovine spongioform encephalopathy. Further- 
more, familial or genetic transmissible spongioform encephalopathies (gTSEs), including familial/genetic CJD (gCJD), fatal familial insomnia, and GerstmannSträussler-Scheinker disease can be distinguished. In gTSEs 30 mutations in the prion protein gene (PRNP) have been demonstrated, many of them being very rare or restricted to specific populations [47]. The physiological function of $\mathrm{PrP}^{\mathrm{C}}$, the normal form of prion protein, is still unclear. The causative agent of TSEs is $\mathrm{PrP}^{\mathrm{SC}}$, a misfolded, protease-resistant version of the normal $\mathrm{PrP}^{\mathrm{C}} \cdot \mathrm{PrP}^{\mathrm{SC}}$ forms aggregates often progressing into large extracellular deposits, described as brain plaques. It is argued that membrane anchoring is an important prerequisite for prion neurotoxicity. Upon prion infection, $\operatorname{PrP}^{\mathrm{C}}$ that is normally anchored to the cell membrane is converted into aggregates of $\mathrm{PrP}^{\mathrm{SC}}$. This might affect signaling of normal membrane-bound $\mathrm{PrP}^{\mathrm{C}}$, leading to neurotoxicity [49].

\section{$\alpha_{1}$-Antitrypsin Deficiency}

$\alpha_{1}$-Antitrypsin $\left(\alpha_{1}\right.$-AT) deficiency is an autosomal recessive disorder that is associated with pulmonary emphysema and may lead to liver cirrhosis. $\alpha_{1}-\mathrm{AT}$ is a small glycoprotein, which is produced in the liver and secreted into the blood. Depending on the allelic variant, the $\alpha_{1^{-}}$ AT serum level is only $10-60 \%$ of normal levels [50]. Its major function is the inhibition of serine proteases, particularly elastase which is released from neutrophil granulocytes, but also of trypsin, chymotrypsin and thrombin. The balance of proteases and protease inhibitors is important. In the lung the 'loss of function' of abnormal $\alpha_{1}$-AT leads to hyperactivity of elastase and proteolytic destruction of structural proteins of the extracellular matrix, resulting in emphysema. The liver damage is caused by the aggregation of the mutated protein [51]. Over 70 naturally occurring variants of $\alpha_{1}$-AT deficiency have been described. The commonest deficiency variants are $S$ and $Z$, resulting from point mutations in the gene. The normal active protein undergoes a conformational transition to inhibit its target proteinase, neutrophil elastase. Due to the $\mathrm{Z}$ mutation the $\alpha_{1}$-AT molecule undergoes conformational change causing an unstable protein that favors dimer formation. This can be further extended to form polymers that tangle in the ER of the hepatocyte to form PAS-positive diastase-resistant inclusion bodies (fig. 3d) [52]. Hepatocyte injury may comprise ballooning liver cell degeneration, chronic hepatitis, cholestasis and liver cirrhosis [51]. $\alpha_{1}$-AT deficiency is also associated with hepatocellular carcinoma [53].

How a Cell Deals with Abnormal

Proteins

\section{Alcoholic and Nonalcoholic Steatohepatitis and} Other Liver Diseases Associated with Mallory Body

\section{Formation}

Deposition of misfolded proteins as cytoplasmic aggregates is also a key feature of several chronic liver diseases, particularly alcoholic and nonalcoholic steatohepatitis (NASH) [54-57]. Alcoholic steatohepatitis (ASH) is a severe necroinflammatory liver disorder, linking the still reversible fatty liver with irreversible liver cirrhosis. Morphologic hallmarks are macro- and microvesicular steatosis, ballooning of hepatocytes, liver cell degeneration, necrosis and apoptosis, pericellular fibrosis, granulocytic inflammation, and disturbance (diminution) of the keratin intermediate filament (IF) cytoskeleton, particularly in ballooned hepatocytes $[54,55,57]$. Ballooning is often associated with the appearance of irregularly shaped cytoplasmic inclusions, termed Mallory bodies (MBs) or alcoholic hyalin (figs. 4, 5). Another common liver disorder with similar morphology is NASH (fig. 4), which represents the hepatic manifestation of the metabolic syndrome and seems to be responsible for a considerable percentage of liver cirrhosis in the western world. This disease is closely associated with obesity, insulin resistance, type II diabetes, hypertension and hyperlipidemia $[54,56]$.

MBs, however, are not ASH- and NASH-specific and can also occur in chronic cholestasis (e.g., primary biliary cirrhosis), copper toxicity (e.g. Wilson's disease, idiopathic copper toxicosis), drug toxicity, and hepatocellular tumors (fig. 5) [58].

Major morphologic features of human ASH or NASH, including MBs, can be reproduced under standardized conditions in mice by chronic intoxication with the porphyrogenic agents griseofulvin [59] or 3,5-diethoxycarbonyl-1,4-dihydrocollidine (DDC) [60]. These models are, therefore, not only valuable to study the pathogenesis of cellular alterations of ASH and NASH but may also contribute to our understanding of mechanisms involved in the development of protein aggregation diseases in general.

MBs of human or mouse origin contain abnormal partially degraded and hyperphosphorylated keratins, particularly misfolded keratin (K) 8 (fig. 4b), ubiquitin (fig. 4d) and p62 (fig. 4c) as major, and Hsp25 and Hsp70 as minor components, as shown by immunohistochemistry and chemical and immunochemical analyses [44, 58]. Structural analysis of MBs using infrared spectroscopy revealed a $\beta$-sheet conformation. The relative amount of $\beta$-sheets in MBs was increased compared to $\alpha$-helices, indicating a conformational change in the $\mathrm{K}$ peptides [61]. In this context p62 could be protective by 


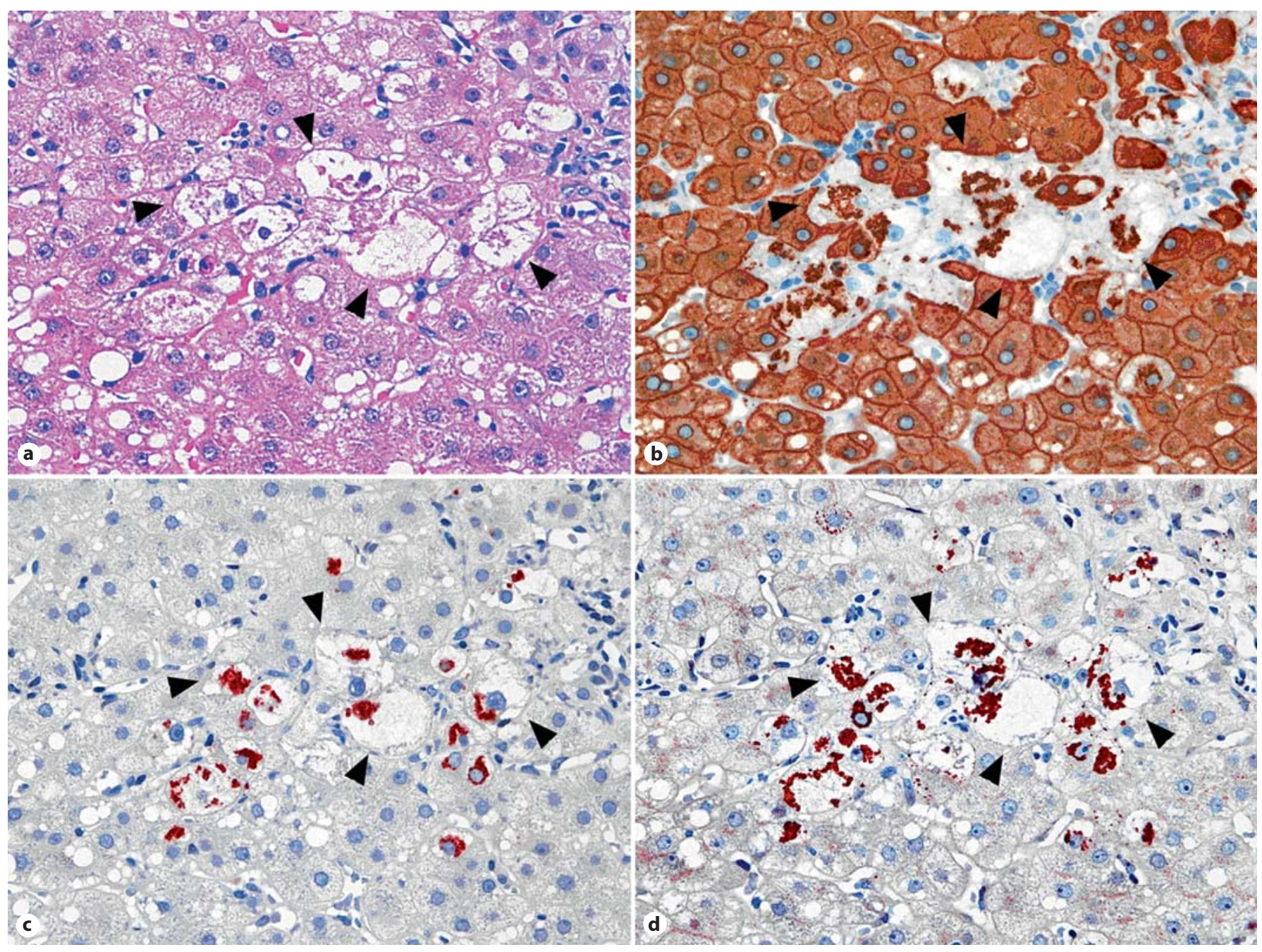

Fig. 4. NASH. a Note enlargement and ballooning of hepatocytes containing MBs (arrowheads). HE. b The ballooned hepatocytes remain unstained with keratin antibodies and only MBs show keratin immunoreactivity (arrowheads). Immunostaining with p62 (c) and ubiquitin (d) antibodies reveal decoration of MBs. Arrowheads indicate MB-containing cells. a-d $\times 400$.

sequestration of abnormal proteins as inert but potentially reversible inclusion bodies, particularly if proteolysis is impaired [58].

Mice treated with DDC for different time periods allow monitoring under well-defined conditions the time course of $\mathrm{MB}$ formation. The results of these studies revealed two essential steps, namely increase of $\mathrm{p} 62 \mathrm{mRNA}$ and protein and $\mathrm{K} 8$ and $\mathrm{K} 18$ overexpression and hyperphosphorylation concomitant with $\mathrm{K}$ cytoskeleton derangement. In the later stages of intoxication the $1: 1$ ratio of $\mathrm{K} 8: \mathrm{K} 18$, which is essential for IF formation, is disturbed and the balance is shifted towards K8. To elucidate the role of different components in $\mathrm{MB}$ formation and related cytoskeletal damage, knockout mice with deletion of $\mathrm{K} 8$ or K18 were studied $[62,63]$. In the absence of either K8 (K8-/-) or K18 (K18-/-) no IFs could be assembled and the remaining 'widowed' K partner was degraded. DDC intoxication of K-deleted animals revealed that MBs did not form in K8-/- mice despite an abundance of p62. This demonstrates that $\mathrm{K} 8$ is the core component of $\mathrm{MB}$ formation [63]. The findings that $\mathrm{K}$ overexpression and phosphorylation occur upon DDC intoxication and the fact that K8-/mice have an increased sensitivity to toxins suggest that K8 plays a protective role $[63,64]$. The clinical relevance of this finding is supported by recent observations that some patients with 'idiopathic' liver cirrhosis have mutations in 
the K8 gene $[65,66]$. Is this increased resistance to toxins in wild-type mice related to an intact cytoskeleton responsible for stability of hepatocytes or does it reflect a noncytoskeletal function of $\mathrm{K} 8$ ? The answer is given by the behavior of K18-/- mice. These mice produce only K8 and resist intoxication similar to wild-type animals despite the lack of a hepatocellular IF cytoskeleton. Moreover, these animals develop MBs consisting of K8 and p62 already after short-term DDC intoxication (unpubl. observation) and also spontaneously at old age [62]. Since at least in vitro transfected $\mathrm{K} 8$ is readily degraded by the proteasomal system after ubiquitination [67] this finding is indirect evidence for the impairment of proteasomal action in conditions associated with $\mathrm{MB}$ formation. Furthermore, MB formation can be induced by proteasomal inhibition. This has been shown in primary tissue cultures of hepatocytes from normal and drug-primed MB-forming livers, which were incubated with the proteasome inhibitor PS-341 [68].

The protein p62 is an immediate early response gene product. In tissue culture cells, p62 transcript and protein rapidly increase in response to a variety of signals for proliferation, differentiation, and particularly to oxidative stress. p62 acts as an adapter protein in the NF- $\kappa B$ pathway and could therefore be essential in the context of the cellular reactions to oxidative stress since NF- $\mathrm{KB}$ is a major mediator in this respect $[69,70]$. Moreover, due to its affinity to polyubiquitinated abnormal proteins that arise in higher quantities during stress, it is possible that p62 also interacts with the other polyubiquitin-dependent cellular systems, particularly the proteasome. Indeed, p62 seems to shuttle ubiquitinated proteins to the proteasome [71]. p62 itself, if overexpressed, shows a tendency to aggregate as intracellular hyaline bodies which may be present alone or together with MBs or even in close association with MBs as hybrid inclusions [72].

The presence of p62 together with other stress proteins, such as Hsp70, Hsp25, and ubiquitin, in MBs not only points to oxidative stress as key player in their pathogenesis, but indicates that particularly in chronic stress situations segregation and aggregation constitute a third line of defense against abnormal misfolded proteins.

Fig. 5. Hepatocellular carcinoma. a Arrow indicates MB in a tumor cell. HE. b Immunohistochemical staining for p62 in a tumor cell. Arrow indicates p62 as component of MB in a tumor cell. c Electron microscopy shows the filamentous ultrastructure of the MB. a, b $\times 1,500$. c Bar: $250 \mathrm{~nm}$.

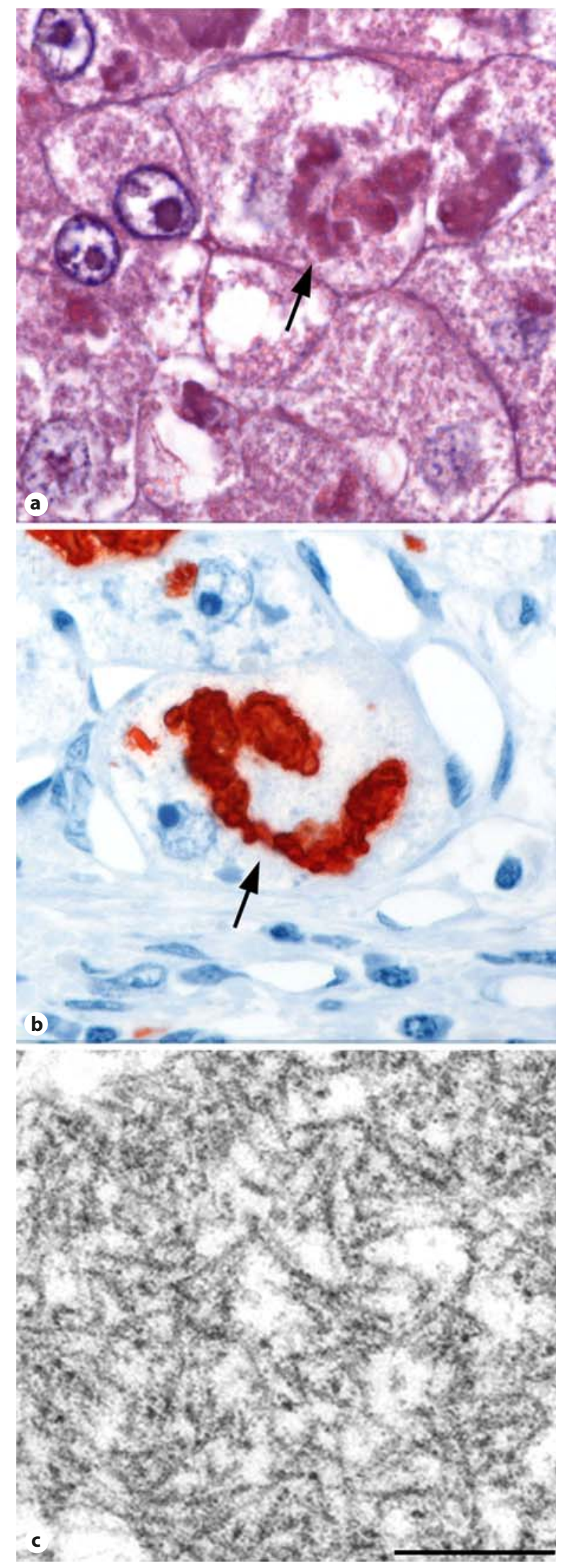

Pathobiology 2007;74:145-158

How a Cell Deals with Abnorma 
Thereby p62 could act as reservoir for altered proteins and prevent their irreversible aggregation or proteolytic destruction by keeping them in a folding-competent state until refolding is possible after recovery from stress. The interplay between its function in signal transduction and sequestosome formation, although likely, is as yet unclear and needs further attention [44].

How can the development of IF cytoskeletal alterations concomitant with $\mathrm{MB}$ formation be explained? Destruction of the IF cytoskeleton can ensue from disassembly of IFs due to (hyper)phosphorylation and/or partial proteolysis (truncation) of $\mathrm{K}$ subunits, impaired IF assembly due to altered $\mathrm{K}$ subunits, or disturbed subunit stoichiometry with prevalence of $\mathrm{K} 8$, which has been shown to occur in DDC-intoxicated mice [63].

On the basis of studies on MB development in human and mouse livers the scenario leading to cytoskeletal alterations and $\mathrm{MB}$ formation in chronically stressed livers can be summarized as follows [64]. (1) In the early stage increased synthesis of $\mathrm{K} 8$ and $\mathrm{K} 18$ occurs resulting in increased density of the IF cytoplasmic network. (2) Thereafter IF assembly is hampered by (hyper)phosphorylation of K subunits. (3) In still later stages of intoxication K8 prevails over K18 due to unequal synthesis and/or decreased degradation favoring a shift from the filamentous to the nonfilamentous state with reduction of the IF cytoskeleton. It is interesting in this context that K8 is a preferred substrate of transglutaminase and could be stabilized by cross-linking in contrast to K18 [73]. (4) Phosphorylated K8 subunits not accompanied by K18 are ubiquitinated and finally degraded by the proteasomal machinery. (5) In chronic stress situations, however, proteolysis is impaired and the degradation cannot keep up with the production of abnormal ubiquitinated Ks, particularly K8, which finally aggregate as MBs together with p62 and are stabilized by transglutaminase-mediated cross-linking [74] (fig. 2).

A similar scenario might be valid for other inclusion body (protein aggregation) diseases, where instead of keratins other cellular proteins undergo misfolding, like chronic degenerative disorders of the central nervous system.

These findings raise the question whether inclusions in a cell are only markers of a special type of injury or injurious by themselves? No general answers can be given. It is likely that the nature of the aggregated protein determines their biological significance. So far there is no clear-cut evidence for the toxicity of MBs in hepatocytes, whereas in neuronal cells protein aggregates seem to interfere with microtubule-dependent axonal transport.
Toxicity of aggregates could also result from sequestering, and thus functional unavailability, of essential cellular components, from overwhelming the capacity of the chaperone machinery, or paralysis of the ubiquitin-proteasome pathway by adhering indigestible ubiquitinated aggregates. As a consequence, proteasomes would be unable to degrade other substrates, such as transcription factors and cyclins, essential for cell survival. Thus, many molecular events involved in the pathogenesis and the effects of protein inclusion bodies in a living cell still remain to be investigated.

\section{Therapeutic Implications}

Detailed knowledge of protein folding and misfolding mechanisms offers new options regarding targeted therapies under the assumption that inclusion bodies may be toxic to the cell, which may not always be the case. Possible targets for therapy are reducing protein misfolding and blocking aggregation. Strategies include (1) blocking the expression or accumulation of the abnormal protein, (2) inhibiting the tendency of the protein to aggregate, (3) enhancing chaperone expression, (4) overexpressing E3 ligases, (5) enhancing expression of shuttling proteins, such as p62, and (6) upregulating proteasome activity. Obviously, an interactive approach will be needed for prevention and therapy of protein aggregation diseases $[75,76]$.

With respect to neurodegenerative diseases, especially Alzheimer's disease, many new therapies targeting the mechanisms underlying the disease are now in progress. Three strategies have reached phase II clinical trials. All are based on reducing the amount of protofibrillar $A \beta$ aggregation, each involving different strategies. (1) The first is an $A \beta$ vaccine that was tested in mice, which where inoculated with an $A \beta$-derived immunogen, resulting in the reduction of amyloid plaques. In further studies passive immunization by infusion of $A \beta$-directed antibodies had a similar effect. The results led to the hypothesis that the vaccine caused redistribution of $A \beta$ from the brain into the periphery, where it could be degraded. (2) The second strategy involves the inhibition of $A \beta$ aggregation by binding monomeric $A \beta$ with a small molecule that is thought to be a proteoglycan mimic. Tramiprosate (3amino-1-propanesulfonic acid) was found to maintain $A \beta$ in a nonfibrillar form. The compound reduced amyloid-plaque formation in mouse models; in phase II trials some patients showed some improvement in cognitive measures after receiving treatment for more than a year. Phase III trials are underway. (3) The third approach is to reduce the production of Ab42 from APP (and Ab42 aggregation) by modulating $\gamma$-secretase/presenilin activity. 
Several companies have reported that $\gamma$-secretase inhibitors are entering phase II trials [77].

Another interesting opportunity for new therapeutic interactions might be based on the fact that apolipoprotein $\mathrm{E}$ (ApoE) $\varepsilon 4$ allele of the ApoE gene has emerged as a major genetic risk factor for Alzheimer's disease. The two domains of apoE interact more strongly with each other in apoE4 than in the other apoE isoforms, which may account for the apoE4's adverse effects. Compounds that disrupt this interaction are being developed. Another molecule that may be a potential target is the microtubule-associated protein tau, the major component of neurofibrillar tangles. Tau kinase inhibitors seem to be a potential treatment, because tau phoshorylation increases dramatically in Alzheimer's disease. Furthermore, tau aggregation can be blocked either directly or by inhibiting its proteolysis. Hyperphosphorylated tau dissociates easily from microtubules, reducing their stability. Therefore microtubule-stabilizing drugs could be an interesting option [78].

\section{Acknowledgements}

We thank Andrea Kaps and Herwig Brandtner for their skillful assistance in photograph and figure design.

\section{References}

1 Dobson CM: Principles of protein folding, misfolding and aggregation. Semin Cell Dev Biol 2004;15:3-16.

$\checkmark 2$ Dobson CM: Protein folding and misfolding Nature 2003;426:884-890.

-3 Uversky VN: Protein folding revisited. A polypeptide chain at the folding-misfoldingnonfolding cross-roads: which way to go? Cell Mol Life Sci 2003;60:1852-1871.

$\checkmark 4$ Kuznetsov G, Nigam SK: Folding of secretory and membrane proteins. $\mathrm{N}$ Engl J Med 1998:339:1688-1695.

5 Garcia-Mata R, Gao YS, Sztul E: Hassles with taking out the garbage: aggravating aggresomes. Traffic 2002;3:388-396.

-6 Barral JM, Broadley SA, Schaffar G, Hartl FU: Roles of molecular chaperones in protein misfolding diseases. Semin Cell Dev Biol 2004; $15: 17-29$

7 Wickner S, Maurizi MR, Gottesman S: Posttranslational quality control: folding, refolding, and degrading proteins. Science 1999; 286:1888-1893.

8 Horwich A: Cell biology: sight at the end of the tunnel. Nature 2004;431:520-522.

-9 Kopito RR: Aggresomes, inclusion bodies and protein aggregation. Trends Cell Biol 2000;10:524-530.

10 Shin J: P62 and the sequestosome, a novel mechanism for protein metabolism. Arch Pharm Res 1998;21:629-633.

-11 Csermely P, Schnaider T, Soti C, Prohaszka Z, Nardai G: The 90-kDa molecular chaperone family: structure, function, and clinical applications. A comprehensive review. Pharmacol Ther 1998;79:129-168.

12 Soti C, Sreedhar AS, Csermely P: Apoptosis, necrosis and cellular senescence: chaperone occupancy as a potential switch. Aging Cell 2003;2:39-45.

13 Carver JA, Rekas A, Thorn DC, Wilson MR: Small heat-shock proteins and clusterin: intra- and extracellular molecular chaperones with a common mechanism of action and function? IUBMB Life 2003;55:661-668.
4 Hayes SA, Dice JF: Roles of molecular chaperones in protein degradation. J Cell Biol 1996;132:255-258.

15 McClellan AJ, Tam S, Kaganovich D, Frydman J: Protein quality control: chaperones culling corrupt conformations. Nat Cell Biol 2005; 7:736-741.

-16 Sherman MY, Goldberg AL: Cellular defenses against unfolded proteins: a cell biologist thinks about neurodegenerative diseases. Neuron 2001;29:15-32.

$\checkmark 17$ Soti C, Csermely P: Aging and molecular chaperones. Exp Gerontol 2003;38:10371040.

18 Mosser DD, Morimoto RI: Molecular chaperones and the stress of oncogenesis. Oncogene 2004;23:2907-2918.

19 Gregersen N, Bolund L, Bross P: Protein misfolding, aggregation, and degradation in disease. Mol Biotechnol 2005;31:141-150.

-20 Poon S, Easterbrook-Smith SB, Rybchyn MS, Carver JA, Wilson MR: Clusterin is an ATP independent chaperone with very broad substrate specificity that stabilizes stressed proteins in a folding-competent state. Biochemistry 2000;39:15953-15960.

21 Grune T, Jung T, Merker K, Davies KJ: Decreased proteolysis caused by protein aggregates, inclusion bodies, plaques, lipofuscin, ceroid, and 'aggresomes' during oxidative stress, aging, and disease. Int J Biochem Cell Biol 2004;36:2519-2530.

22 Berke SJ, Paulson HL: Protein aggregation and the ubiquitin proteasome pathway: gaining the UPPer hand on neurodegeneration. Curr Opin Genet Dev 2003;13:253-261.

23 Glickman MH: Getting in and out of the proteasome. Semin Cell Dev Biol 2000;11:149158.

24 Pines J, Lindon C: Proteolysis: anytime, any place, anywhere? Nat Cell Biol 2005;7:731735.

25 Elsasser S, Finley D: Delivery of ubiquitinated substrates to protein-unfolding machines. Nat Cell Biol 2005;7:742-749.
26 Goldberg AL: Protein degradation and protection against misfolded or damaged proteins. Nature 2003;426:895-899.

27 Johnston JA, Ward CL, Kopito RR: Aggresomes: a cellular response to misfolded proteins. J Cell Biol 1998;143:1883-1898.

28 Johnston JA, Illing ME, Kopito RR: Cytoplasmic dynein/dynactin mediates the assembly of aggresomes. Cell Motil Cytoskeleton 2002;53:26-38.

29 Sunde M, Blake CC: From the globular to the fibrous state: protein structure and structural conversion in amyloid formation. Q Rev Biophys 1998;31:1-39.

30 Merlini G, Bellotti V: Molecular mechanisms of amyloidosis. N Engl J Med 2003; 349:583-596.

- 31 Carulla N, Caddy GL, Hall DR, Zurdo J, Gairi M, Feliz M, Giralt E, Robinson CV, Dobson CM: Molecular recycling within amyloid fibrils. Nature 2005;436:554-558.

32 Selkoe DJ: Folding proteins in fatal ways. Nature 2003;426:900-904.

33 Falk RH, Comenzo RL, Skinner M: The systemic amyloidoses. N Engl J Med 1997;337: 898-909.

34 Bataille R, Harousseau JL: Multiple myeloma. N Engl J Med 1997;336:1657-1664.

35 Freemont AJ: The pathology of dialysis. Semin Dial 2002;15:227-231.

36 Rowland LP, Shneider NA: Amyotrophic lateral sclerosis. N Engl J Med 2001;344:16881700.

37 Martin JB: Molecular basis of the neurodegenerative disorders. N Engl J Med 1999;340: 1970-1980.

38 Soto C: Unfolding the role of protein misfolding in neurodegenerative diseases. Nat Rev Neurosci 2003;4:49-60.

39 Ross CA, Poirier MA: Protein aggregation and neurodegenerative disease. Nat Med 2004;10(suppl):S10-S17.

40 Janig E, Stumptner C, Fuchsbichler A, Denk $\mathrm{H}$, Zatloukal K: Interaction of stress proteins with misfolded keratins. Eur J Cell Biol 2005; 84:329-339. 
41 Choi-Miura NH, Oda T: Relationship between multifunctional protein 'clusterin' and Alzheimer disease. Neurobiol Aging 1996;17:717-722.

-42 Selkoe DJ: Cell biology of protein misfolding: the examples of Alzheimer's and Parkinson's diseases. Nat Cell Biol 2004;6:1054-1061.

43 Kuusisto E, Salminen A, Alafuzoff I: Early accumulation of p62 in neurofibrillary tangles in Alzheimer's disease: possible role in tangle formation. Neuropathol Appl Neurobiol 2002;28:228-237.

- 44 Zatloukal K, Stumptner C, Fuchsbichler A, Heid H, Schnoelzer M, Kenner L, Kleinert R, Prinz M, Aguzzi A, Denk H: p62 is a common component of cytoplasmic inclusions in protein aggregation diseases. Am J Pathol 2002;160:255-263.

-45 Nakano T, Nakaso K, Nakashima K, Ohama E: Expression of ubiquitin-binding protein p62 in ubiquitin-immunoreactive intraneuronal inclusions in amyotrophic lateral sclerosis with dementia: analysis of five autopsy cases with broad clinicopathological spectrum. Acta Neuropathol (Berl) 2004;107: 359-364.

-46 Nagaoka U, Kim K, Jana NR, Doi H, Maruyama M, Mitsui K, Oyama F, Nukina $\mathrm{N}$ : Increased expression of $\mathrm{p} 62$ in expanded polyglutamine-expressing cells and its association with polyglutamine inclusions. J Neurochem 2004;91:57-68.

- 47 Kovacs GG, Puopolo M, Ladogana A, Pocchiari M, Budka H, van Duijn C, Collins SJ, Boyd A, Giulivi A, Coulthart M, DelasnerieLaupretre N, Brandel JP, Zerr I, Kretzschmar HA, de Pedro-Cuesta J, Calero-Lara M, Glatzel M, Aguzzi A, Bishop M, Knight R, Belay G, Will R, Mitrova E: Genetic prion disease: the EUROCJD experience. Hum Genet 2005; 118:166-174.

-48 Aguzzi A, Miele G: Recent advances in prion biology. Curr Opin Neurol 2004;17:337342.

49 Aguzzi A: Cell biology. Prion toxicity: all sail and no anchor. Science 2005;308:14201421.

-50 Crowther DC, Belorgey D, Miranda E, Kinghorn KJ, Sharp LK, Lomas DA: Practical genetics: alpha-1-antitrypsin deficiency and the serpinopathies. Eur J Hum Genet 2004; 12:167-172.

51 Kumar V, Cotran RS, Robbins SL: Basic Pathology, ed 7. Philadelphia, Saunders, 2002.

-52 Lomas DA, Parfrey H: Alpha1-antitrypsin deficiency. 4. Molecular pathophysiology. Thorax 2004;59:529-535.
53 Parfrey H, Mahadeva R, Lomas DA: Alpha(1)-antitrypsin deficiency, liver disease and emphysema. Int $\mathrm{J}$ Biochem Cell Biol 2003;35:1009-1014.

54 Denk H, Stumptner C, Fuchsbichler A, Zatloukal K: Alcoholic and nonalcoholic steatohepatitis. Histopathologic and pathogenetic considerations. Pathologe 2001;22:388-398.

55 Brunt EM: Alcoholic and nonalcoholic steatohepatitis. Clin Liver Dis 2002;6:399-420, vii.

56 Brunt EM: Nonalcoholic steatohepatitis. Semin Liver Dis 2004;24:3-20.

57 Brunt EM, Tiniakos DG: Pathology of steatohepatitis. Best Pract Res Clin Gastroenterol 2002;16:691-707

-58 Stumptner C, Fuchsbichler A, Heid H, Zatloukal K, Denk H: Mallory body - a diseaseassociated type of sequestosome. Hepatology 2002;35:1053-1062.

59 Denk H, Gschnait F, Wolff K: Hepatocellular hyalin (Mallory bodies) in long term griseofulvin-treated mice: a new experimental model for the study of hyalin formation. Lab Invest 1975;32:773-776.

60 Tsunoo C, Harwood TR, Arak S, Yokoo H Cytoskeletal alterations leading to Mallory body formation in livers of mice fed 3,5diethoxycarbonyl-1,4-dihydrocollidine. Hepatol 1987;5:85-97.

61 Kachi K, Wong PT, French SW: Molecular structural changes in Mallory body proteins in human and mouse livers: an infrared spectroscopy study. Exp Mol Pathol 1993;59: 197-210.

62 Magin TM, Schröder R, Leitgeb S, Wanninger F, Zatloukal K, Grund C, Melton DW: Lessons from keratin 18 knockout mice: formation of novel keratin filaments, secondary loss of keratin 7 and accumulation of liverspecific keratin 8-positive aggregates. J Cell Biol 1998;140:1441-1451.

63 Zatloukal K, Stumptner C, Lehner M, Denk H, Baribault H, Eshkind LG, Franke WW: Cytokeratin 8 protects from hepatotoxicity, and its ratio to cytokeratin 18 determines the ability of hepatocytes to form Mallory bodies. Am J Pathol 2000;156:1263-1274.

64 Denk H, Stumptner C, Zatloukal K: Mallory bodies revisited. J Hepatol 2000;32:689702.

$65 \mathrm{Ku}$ NO, Gish R, Wright TL, Omary MB: Keratin 8 mutations in patients with cryptogenic liver disease. N Engl J Med 2001;344:15801587.
$66 \mathrm{Ku}$ NO, Darling JM, Krams SM, Esquivel CO, Keeffe EB, Sibley RK, Lee YM, Wright TL, Omary MB: Keratin 8 and 18 mutations are risk factors for developing liver disease of multiple etiologies. Proc Natl Acad Sci USA 2003; 100:6063-6068.

$67 \mathrm{Ku}$ NO, Omary MB: Keratins turn over by ubiquitination in a phosphorylation-modulated fashion. J Cell Biol 2000;149:547-552.

68 Bardag-Gorce F, Riley NE, Nan L, Montgomery RO, Li J, French BA, Lue YH, French SW: The proteasome inhibitor, PS-341, causes cytokeratin aggresome formation. Exp Mol Pathol 2004;76:9-16.

69 Heck S, Lezoualc'h F, Engert S, Behl C: Insulin-like growth factor-1-mediated neuroprotection against oxidative stress is associated with activation of nuclear factor kappaB. J Biol Chem 1999;274:9828-9835.

70 Mattson MP, Culmsee C, Yu Z, Camandola $S$ : Roles of nuclear factor kappa B in neuronal survival and plasticity. J Neurochem 2000; 74:443-456.

-71 Seibenhener ML, Babu JR, Geetha T, Wong HC, Krishna NR, Wooten MW: Sequestosome $1 /$ p 62 is a polyubiquitin chain binding protein involved in ubiquitin proteasome degradation. Mol Cell Biol 2004;24:80558068.

72 Denk H, Stumptner C, Fuchsbichler A, Muller T, Farr G, Muller W, Terracciano L, Zatloukal K: Are the Mallory bodies and intracellular hyaline bodies in neoplastic and non-neoplastic hepatocytes related? J Pathol 2006;208:653-661.

73 Zatloukal K, Denk H, Lackinger E, Rainer I: Hepatocellular cytokeratins as substrates of transglutaminases. Lab Invest 1989;61:603608

74 Zatloukal K, Fesus L, Denk H, Tarcsa E, Spurej G, Böck G: High amount of epsilon(gamma-glutamyl)lysine cross-links in Mallory bodies. Lab Invest 1992;66:774-777.

-75 Wooten MW, Hu X, Babu JR, Seibenhener ML, Geetha T, Paine MG, Wooten MC: Signaling, polyubiquitination, trafficking, and inclusions: sequestosome 1/p62's role in neurodegenerative disease. J Biomed Biotechnol 2006:2006:62079

76 Taylor JP, Hardy J, Fischbeck KH: Toxic proteins in neurodegenerative disease. Science 2002;296:1991-1995.

2002;296:1991-1995.
77 Lansbury PT, Lashuel HA: A century-old debate on protein aggregation and neurodegeneration enters the clinic. Nature 2006; 443:774-779.

78 Roberson ED, Mucke L: 100 years and counting: prospects for defeating Alzheimer's disease. Science 2006;314:781-784 